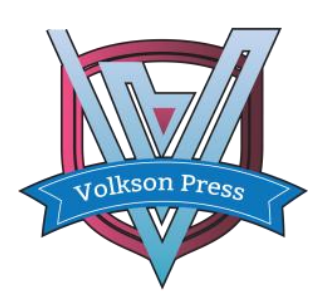

Contents List available at VOLKSON PRESS

Mechanical and Control Engineering (MCE)

DOI : http://doi.org/10.26480/wsmce.01.2017.46.47

\title{
RESEARCH AND DEVELOPMENT OF STATIC LOAD TEST BENCH FOR EMU GEAR UNIT
}

\author{
Cao Yu*, Li Zihua, Dong Bo \\ China Railway Test \& Certification Centre, Jiuxianqiao North Road 1, Beijing, China \\ *Corresponding Author: chycrcc@163.com
}

This is an open access article distributed under the Creative Commons Attribution License, which permits unrestricted use, distribution, and reproduction in any medium, provided the original work is properly cited

\section{ARTICLE DETAILS}

\section{Article History:}

Received 02 october 2017

Accepted 06 october 2017

Available online 11 november 2017

Keywords

Test bench, EMU gear unit, Static load test.

\section{ABSTRACT}

The gear unit has been recently added to the list of the railway products that require compulsory certification according to China Railway Corporation. The test of the gear unit becomes much more important and urgent. It is summarized in this paper that a new static load test bench for EMU gear unit is researched and developed. The static load test of EMU gear unit is expected to be carried out efficiently with this test bench. Practicality of the test bench is validated, and it turns out that it is compact, highly efficient, labor-saving, of higher structural strength, suitable for various types of EMU gear unit and is of simple operation, which provides a strong support for the improvements of EMU gear unit design.

\section{Introduction}

China's high-speed railway is in the golden age of development [1]. The performance of the traction drive systems directly affects that of the bogies, which is related to the safety of the railway system. And it is vital that the operation of the gear unit is reliable and steady, as it is the core component of the traction drive system. On the other hand, according to China Railway Corporation, the gear unit has been added to the list of the railway products that require compulsory certification. Therefore, the test of the gear unit becomes much more important and urgent [2].

\section{CURRENT SITUATION OF THE TEST}

The technology of high-speed gear is studied since 1970s in China. And the manufacturing process is not as developed as it is abroad. The current supply of high-speed EMU gear unit is dominated by foreign manufacturers, including Voith, Flanders, ZF, etc. This situation leads to defects of technology for gear unit's test, seriously restricting the independent development of China's high-speed train.

The gear units produced by domestic manufacturers are so simply tested before leaving factories that it does not meet the certification requirements made by China Railway Corporation. Therefore, it is necessary for quality inspection agencies to study the technology of the EMU gear unit test in depth, in order to improve the domestic independent development of the EMU gear unit design.

In this paper, the technical standard of the static load test of the EMU gear unit is researched, and a new static load test bench for EMU gear unit is developed.

\section{TEST REQUIREMENTS}

According to TJ/CL 277 EMU Gear Unit Provisional Technical Conditions, the gear unit consists of gearbox housing, gears, bearings, seals, connection components between gearbox and bogie frame (e.g. Cbracket), fasteners, etc. And the EMU gear unit static load test consists of bracket static strength test and gearbox housing static strength test.

The bracket is subjected to a stress test under the motor short-circuit torque (coupling slip torque) condition, and the measured stress shall not be greater than the nominal yield strength of the bracket material.

The same requirements for the gearbox housing. The gearbox housing is subjected to a stress test under the motor short-circuit torque (coupling slip torque) condition, and the measured stress shall not be greater than the nominal yield strength of the gearbox housing material.

\section{DESIGN SCHEME}

Most of the EMU gear units are coupled with couplings at the end of the input shafts, so according to the requirements of the test, the static test should be carried out under the limited torque of the couplings.

\subsection{Structural design}

The designed static load test bench for EMU gear unit consists of platform, anti-rotation bracket, C-bracket fixture, torque sensor and torque sensor bracket. The anti-rotation bracket, the C-bracket fixture and the torque sensor bracket are mounted on the platform, and their positions can be adjusted according to different sizes of the gear units. The torque senor is coupled to the coupling at the end of the input shaft of the gear unit by the dual flange.

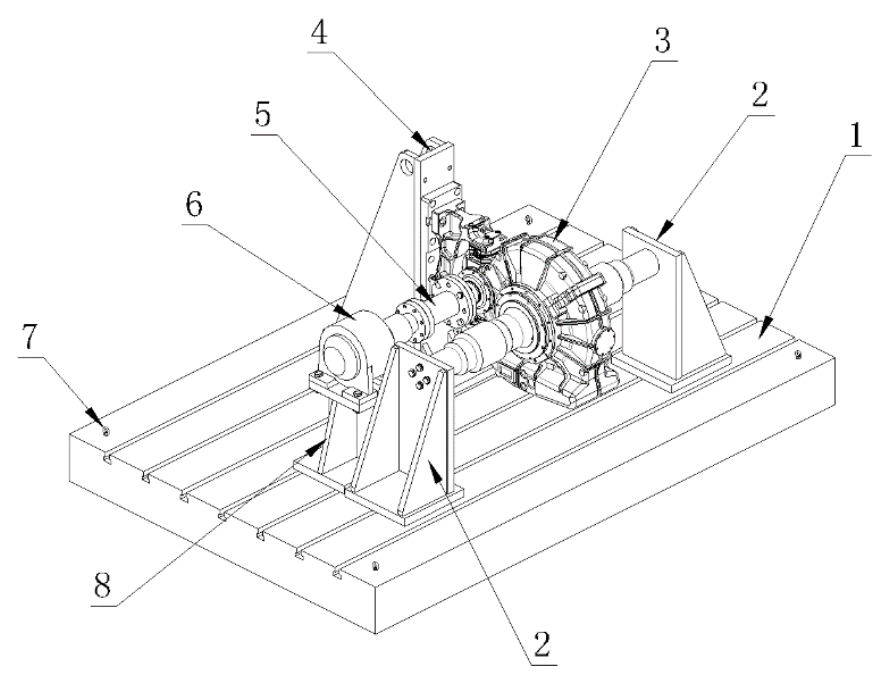

1-Platform, 2-Anti-rotation bracket, 3-Gear unit, 4-C-bracket fixture, 5-Dual flange, 6-Torque sensor, 7-Eye bolt, 8-Torque sensor bracket 
Figure 1: Structure Diagram

As shown in Figure 1, the output shaft of the gear unit is bolted on and supported by two anti-rotation brackets, which keeps the shaft steady and from rotation during the test. The C-bracket of the gear unit is bolted on the C-bracket fixture. There are several sets of screw holes on the Cbracket fixture, where different types of gear units that are of different heights can be bolted. One side of the dual flange is connected to the torque sensor flange with cylindrical pins and the other side is connected to the coupling at the end of the input shaft of the gear unit with cylindrical pins as well. And the torque sensor is bolted on the torque sensor bracket, which is mounted on the platform. The entire test bench is driven by hydraulic system and they are controlled by the host computer. Thus, the interaction between human and machine is built up.

\subsection{Technical characteristics}

There are three main advantages of this static load test bench for EMU gear unit:

1. The structure is compact, the position of the bracket and the fixture can be adjusted, which makes this test bench suitable for different types of gear units.

2. Instead of bolts, cylindrical pins are applied to connect the dual flange with the torque sensor and the gear unit, which is of higher structural strength.

3. The static load is applied by the hydraulic system, which is precise enough to meet the test requirements, and the whole process of the test is monitored in real-time with the computer, which is highly efficient, labor-saving and of simple operation.

\section{VALIDATION}

Practicality of the static load test bench for EMU gear unit is validated by a type of C-bracket. The test was carried out under the coupling slip torque condition [3]. The measured stress of each measuring point shall not be greater than the nominal yield strength of the C-bracket material [4].
Table 1: Technical Parameters of C-bracket

\begin{tabular}{|c|c|c|c|}
\hline $\begin{array}{l}\text { Coupling slip } \\
(\mathrm{Nm})\end{array}$ & $\begin{array}{l}\text { torqueReaction force at C-bracket under coupling slip } \\
\text { torque }(\mathrm{N})\end{array}$ & $\mathrm{P}_{\text {C-bracket material }}$ & $\begin{array}{l}\text { Nominal yield strength } \\
\text { (MPa) }\end{array}$ \\
\hline $7000-10500$ & 92319 & G22NiMoCr5-6 & 825 \\
\hline
\end{tabular}

The input torque is applied forward and reverse and slowly increases unti a torque of $10500 \mathrm{Nm}$ is reached and the test is repeated 10 times. And the measured stress shall not be greater than the nominal yield strength (825Mpa) of the bracket material (G22NiMoCr5-6) under the maximum coupling slip torque according to the test requirements. The test result turned out that the maximum measured stress is $170.1 \mathrm{MPa}$ when the maximum coupling slip torque is $10500 \mathrm{Nm}$, and that the maximum measured stress is $151.3 \mathrm{MPa}$ when the maximum coupling slip torque is $-10500 \mathrm{Nm}$. With this new static load test bench, the whole process of the test is smooth and safe, and no exception occurs.

\section{CONCLUSION}

The static load test bench for EMU gear unit is researched and developed in this paper. It contributes significantly to the EMU gear unit certification work. Practicality of this static load test bench is validated, and it turns out that it is compact, highly efficient, labor-saving, of higher structural strength, suitable for various types of EMU gear unit and is of simple operation, which provides a strong support for the improvements of EMU gear unit design.

\section{REFERENCES}

[1] Huawu, H. 2007. Development and Technical Innovation of China Railway. Journal of Railway Engineering Society, 24 (7), 1-11.

[2] Wei, J. 2015. Discussion on Risk Control of Product Inspection for Railway Product Certification. Railway Quality Control, 12 (5), 23-24.

[3] Yongshuai, M., Qiliang, W., Feng, G. 2017. Finite element analysis and its test verification on EMU gearbox suspender. Computer Aided Engineering, 26 (4), 27-31.

[4] Hongbo, Q., Siqin, J., Chengpan, W. 2015. Study on High Speed Class EMU Gearbox Test. Locomotive and Rolling Stock Technology, (3), 30-32. 